\title{
3D EBSD Analysis of Hydrogen Induced Cracking in Pipeline Steel Using Serial Section Broad Ion Beam Tomography
}

\author{
M. K. O’Brien ${ }^{1}$, K. O. Findley ${ }^{1}$, T. Hosman² and J. A. Hunt ${ }^{2}$. \\ 1. Colorado School of Mines, Dept. of Metallurgical and Materials Engineering, Golden, CO, USA. \\ 2. Gatan Inc., 5794 W. Las Positas Blvd., Pleasanton, CA 94588 USA.
}

Steels used in "sour service" in the oil and gas industry are subjected to harsh, corrosive internal environments containing high partial pressures of hydrogen sulfide $\left(\mathrm{H}_{2} \mathrm{~S}\right)$. During sour service, the presence of $\mathrm{H}_{2} \mathrm{~S}$ allows for enhanced hydrogen ingress into the steel and subsequent internal cracking, a process called hydrogen induced cracking (HIC). The diffusion of hydrogen atoms and subsequent cracking is affected by the microstructural features present in the steel in two ways: local diffusivity and hydrogen "trapping". The steels utilized for these applications are fine grain thermomechanically processed alloys with complex microstructures that include microconstituents such as polygonal ferrite, quasi-polygonal ferrite, pearlite, martensite/austenite constituents, and carbonitrides precipitates. The current industrial practice is to keep yield strength of sour service pipeline steels low. However, if the role various microstructural features play in crack initiation and propagation events could be elucidated, it is possible that higher strength steels could be developed for use in sour service environments.

The steel of interest in the current study is API X70, which consists of highly substructured non-equiaxed ferrite with martensite/austenite microconstituents no larger than $\sim 5 \mu \mathrm{m}$. Characterizing small microconstituents requires a high-resolution technique and analyzing their effect on cracking requires large spatial scale imaging to acquire the necessary statistics. Three-dimensional analysis is necessary to characterize the crystallographic plane on which cracking occurs.

Tomographic reconstruction of volumes considered large by scanning electron microscopy (SEM) standards $\left(0.01-1 \mathrm{~mm}^{3}\right)$ can be accomplished via serial-section broad-ion-beam tomography (SSBIB) alternately ion-polishing away material with a broad, low-energy noble ion beam and imaging the remaining polished surface with SEM techniques such as EDS or EBSD [1]. Under computer control in the Gatan iPrep SSBIB tool it is practical to acquire EBSD images of hundreds of sections without user intervention and assemble them for 3D (spatial) visualization and measurement [2]. In general, the resulting $3 \mathrm{D}$ images permit significantly better understanding of the structure of complex systems than would be possible with a few strategically acquired 2D images acquired at various depths.

This tomographic reconstruction technique was utilized to characterize HIC damage. The X70 alloy was first charged with hydrogen using NACE Standard TM0284 and sectioned mechanically to locate cracks [3]. The cracked faces were then sectioned using SSBIB, and EBSD and EDS data were collected for each section. 3D reconstructions were then created using DREAM.3D [4] software and imaged using Paraview, an example of which is shown in Figure 1. The crack was then isolated from the 3D reconstruction with plane normals colored according to the inverse pole figure (IPF) coloring scheme, shown in Figure 2. This information is being used to assess the crystallographic planes where cracking occurs both in transgranular and intergranular regions. Initial results indicate that cracking does not occur preferentially on BCC $\{110\}$ slip planes, but rather might prefer planes ranging from $\{001\}$ to $\{112\}$ in the IPF color scheme. 
[1] Winiarski et al., Ultramicroscopy 172, (2017) p52-64.

[2] T. Hosman et al., Microsc. Microanal. 22:3, (2016), p590-1.

[3] NACE Standard TM0284-2003: Evaluation of Pipeline and Pressure Vessel Steels for Resistance to Hydrogen-Induced Cracking, 2003.

[4] Groeber, M.A. \& Jackson, M.A. Integrating Materials (2014) 3: 5.https://doi.org/10.1186/21939772-3-5

[5] The authors acknowledge funding from the Advanced Steel Processing and Products Research Center at Colorado School of Mines.

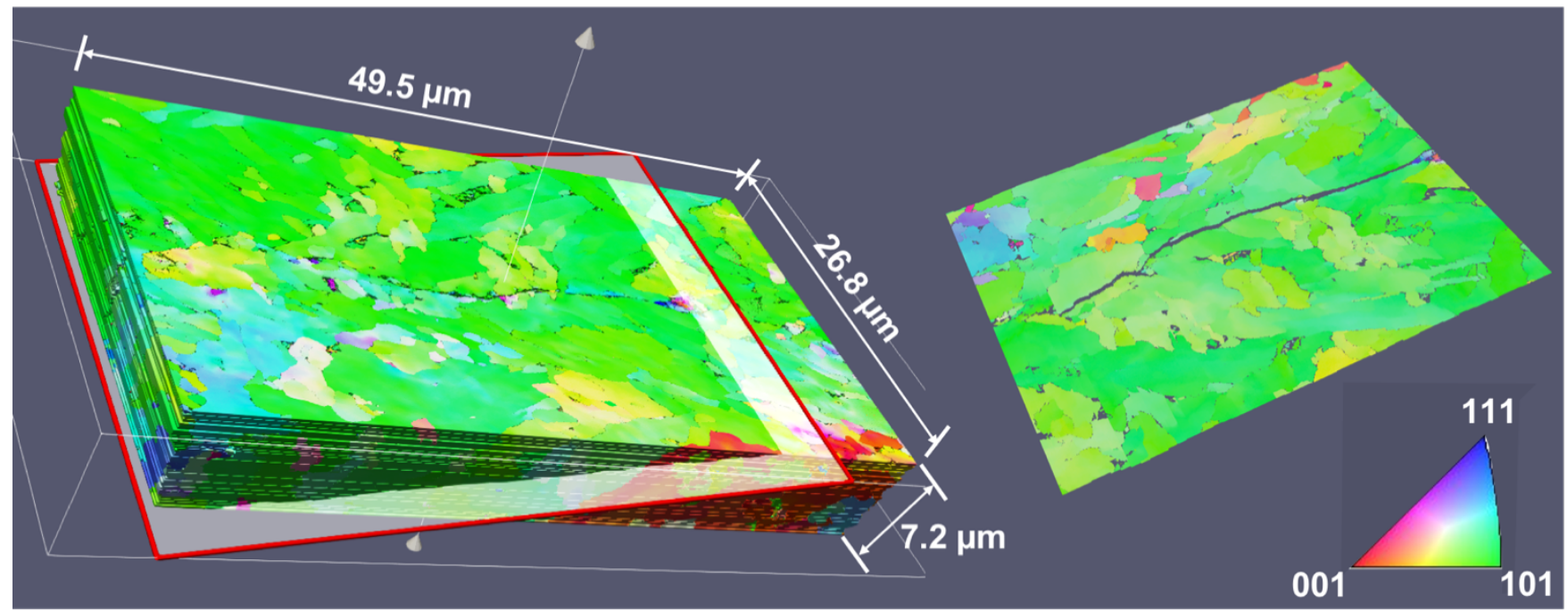

Figure 1. 3D EBSD reconstruction of region near crack tip of hydrogen induced cracking in the X70 steel alloy sectioned using Gatan iPrep SSBIB and colored using IPF coloring.

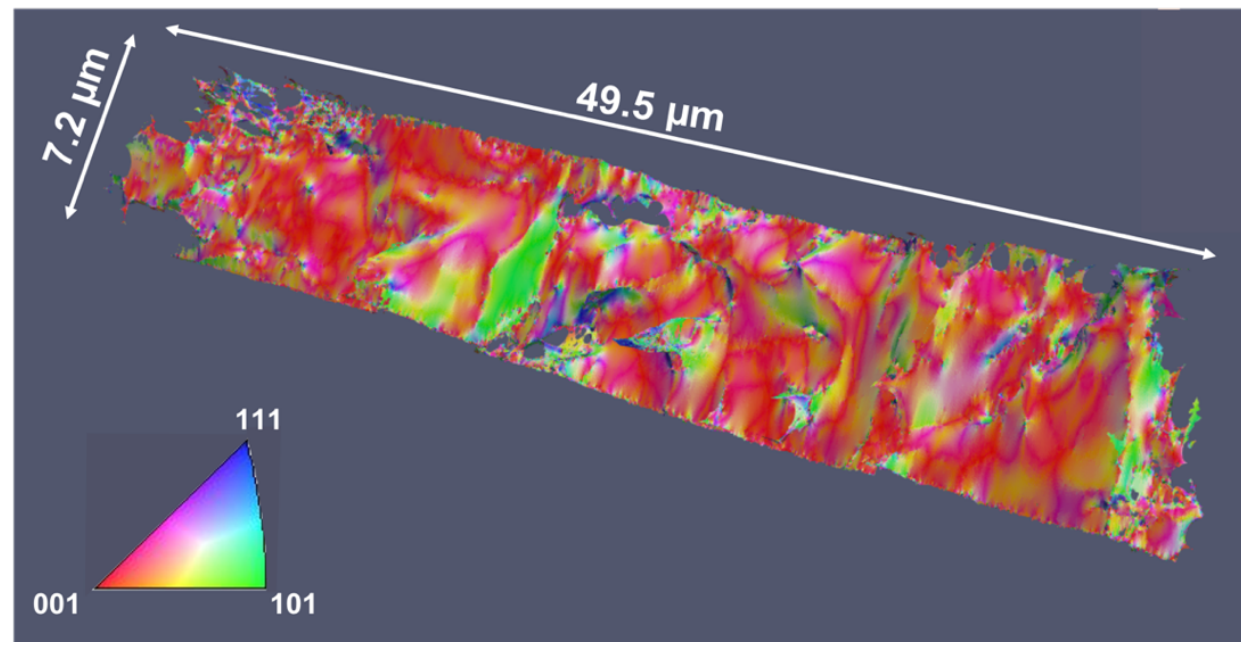

Figure 2. 3D reconstruction of the hydrogen induced crack observed in Figure 1 obtained using Gatan iPrep SSBIB, reconstructed using DREAM.3D, and colored using IPF coloring. 